\title{
Identification of hub genes and pathways in nonalcoholic steatohepatitis by integrated bioinformatics analysis
}

\author{
Pegah Einaliyan ${ }^{1}$, Ali Owfi ${ }^{2}$, Mohammadamin Mahmanzar ${ }^{3,7}$, Taha Aghajanzadeh ${ }^{4,8}$, Morteza \\ Hadizadeh ${ }^{5}$,Ali Sharifi-Zarchi ${ }^{6}$, Behzad Hatami ${ }^{7}$, Hamid Asadzadeh Aghdaei ${ }^{8}$, Mohammad \\ Reza Zali ${ }^{7}$, Kaveh Baghaei ${ }^{7,8 \square}$ \\ ${ }^{1}$ University of Saarland, Germany \\ ${ }^{2}$ Sharif University of Technology, Tehran, Iran \\ ${ }^{3}$ Department of Biophysics, Faculty of Biological Sciences, Tarbiat Modares University, Tehran, Iran \\ ${ }^{4}$ Department of Animal Sciences and Marine Biology, Faculty of Life Sciences and Biotechnology, \\ Shahid Beheshti University, Tehran, Iran \\ ${ }^{5}$ Physiology Research Center, Institute of Basic and Clinical Physiology Sciences, Kerman University of \\ Medical Sciences, Kerman, Iran \\ ${ }^{6}$ Department of Computer Engineering, Sharif University of Technology, Tehran, Iran \\ 7 Gastroenterology and Liver Diseases Research Center, Research Institute for Gastroenterology and \\ Liver Diseases, Shahid Beheshti University of Medical Sciences, Tehran, Iran \\ ${ }^{8}$ Basic and Molecular Epidemiology of Gastrointestinal Disorders Research Center, Research Institute for \\ Gastroenterology and Liver Diseases, Shahid Beheshti University of Medical Sciences, Tehran, Iran \\ Corresponding Author: \\ E-mail: kavehbaghai@gmail.com
}




\begin{abstract}
Background: Currently, non-alcoholic fatty liver disease (NAFLD) is one of the most common chronic liver diseases in the world. Forecasting the short-term, up to 2025, NASH due to fibrosis is one of the leading causes of liver transplantation. Cohort studies revealed that non-alcoholic steatohepatitis (NASH) has a higher risk of fibrosis progression among NAFLD patients. Identifying differentially expressed genes helps to determine NASH pathogenic pathways, make more accurate diagnoses, and prescribe appropriate treatment.

Methods and Results : In this study, we found 11 NASH datasets by searching in the Gene Expression Omnibus (GEO) database. Subsequently, NASH datasets with low-quality control scores were excluded. Four datasets were analyzed with packages of R/Bioconductor. Then, all integrated genes were Imported into Cytoscape to illustrate the protein-protein interactions network. All hubs and nodes degree has been calculated to determine the hub genes with critical roles in networks.Possible correlations between expression profiles of mutual DEGs were identified employing Principal Component Analysis (PCA). Primary analyzed data were filtered based on gene expression ( $\log F C>1, \log F C<-1)$ and adj-P-value $(<0.05)$. Ultimately, among 379 DEGs, we selected the top 10 genes (MYC, JUN, EGR1, FOS, CCL2, IL1B, CXCL8, PTGS2, IL6, SERPINE1) as candidates among up and down regulated genes, and critical pathways such as IL-6, IL-17, TGF $\beta$, and TNF $\alpha$ were identified.
\end{abstract}

\title{
Conclusion:
}

The present study suggests an important DEGs, biological processes, and critical pathways involved in the pathogenesis of NASH disease. Further investigations are needed to clarify the exact mechanisms underlying the development and progression of NASH disease.

Keywords: Differentially expressed genes (DEGs); Non-alcoholic steatohepatitis (NASH); Nonalcoholic fatty liver disease (NAFLD); Protein-protein interaction (PPI); Pathway Analysis; Gene ontology(GO)

Funding: No funding was received to assist with the preparation of this manuscript

Conflicts of interest/Competing interests: The authors have no relevant financial or non-financial interests disclose.

Availability of data and material: The datasets used in this paper are all available on www.ncbi.nlm.nih.gov 


\section{Background:}

The liver is the largest internal organ involved in vital activities such as metabolizing nutrients and energy metabolism, made up of $60 \%$ hepatocyte cells and $40 \%$ non-parenchymal cells [1]. Hepatocytes deficiency in metabolite activity can accelerate non-alcoholic fatty liver disease (NAFLD). Recent studies revealed that among NAFLD patients, non $\square$ alcoholic steatohepatitis (NASH) have a higher risk of fibrosis progression [2,3]. NASH is a significant risk factor for end-stage liver diseases such as cirrhosis and hepatocellular carcinoma (HCC). Statistical studies estimated that up to 2025, NASH-fibrosis is one of the leading causes of liver transplantation [4, 5]. The progression of NASH depends on multifactorial risk factors such as genomic variations, race, obesity, and diabetes. Moreover, many studies suggest that NASH results from several processes like abnormal lipid metabolism, oxidative stress, mitochondrial dysfunction, dysregulation of cytokines and adipokines production. However, the exact mechanism of NASH is not precisely elucidated [5-7].

Several clinical methods detect NAFLD and NASH, such as imaging modalities, blood tests, and biopsy. Also, blood tests, like serum Alanine Aminotransferase (ALT) levels, are commonly used as a surrogate marker of more severe liver status. Liver biopsy is an effective tool to distinguish between simple steatosis and nonalcoholic steatohepatitis. The hallmark of NASH pathological signs are specific cellular features such as necroinflammation, ballooned hepatocytes with or without hepatic fibrosis [2]. However, although biopsy has limitation and risk, liver biopsy remains the gold standard to validate the diagnosis $[2,7]$.

With the development of high-throughput techniques, which several thousand samples are tested simultaneously under given conditions, such as microarray it has become possible to analyze the samples from a proteomic, metabolic and genomic point of view to examine the pathological status of the disease much more accurately and more quickly than before. According to statistics, 20\% of patients with NAFLD develop advanced NASH, and 20\% of NASH patients develop cirrhosis. NASH disease and hepatic fibrosis are known as end-stage disorders, as they are often not diagnosed in early stages. Accordingly, monitoring gene expression levels by high-throughput techniques could help in research with prediction, diagnoses, prognostic and pharmacodynamics for NASH patients, or those at risk for developing NASH to cirrhosis $[8,9]$. Many genomic studies in NAFLD/NASH with concern in genome expression and variations were published to determine pathogenic pathways and genomic variation profiles. Also, gathering and mining biological data at the genomic level and drawing the interaction networks like proteinprotein interaction (PPI) networks have led to understanding and clarifying valuable insights on the NASH pathogenesis and identifying the role of gene expression profiles on the development of NASH. Several bioinformatics studies have demonstrated the potential hub genes, pathways, and mechanisms that play a crucial role in NAFLD progression $[6,10,11]$.

In the present study, we carried out bioinformatics analyses on multiple microarray datasets, including human gene expression profiles from GEO database. Differentially expressed genes (DEGs) in NASH group were identified, and pathway enrichment analysis was performed. In order to find hub genes and GO analysis, we constructed the PPI network of DEGs. Our results suggest the vital role of JUN, IL-6, FOS, CCL2, SERPINE1, IL1B, and MYC in the pathogenesis of NASH disease.

\section{Materials and Methods}

2.1. Data collection The mRNA expression datasets of NASH were searched using the keywords: 'NASH, 'Obesity' and 'Homo sapiens'[porgn: txid9606]', and 'Expression profiling by array' in the Gene Expression Omnibus (GEO) database (http://www.ncbi.nlm.nih.gov/geo) [12]. After a systematic review, eleven GSE profiles 
were selected for primary analysis. While seven GSE profiles, including GSE83452, GSE68421, GSE24807, GSE96971, GSE49012, GSE72756 and GSE134438 were excluded from the study after quality control, four GSE profiles (GSE37031, GSE48452, GSE89632, and GSE17470) were selected for further analyses. GSE37031 was based on GPL14877 (Affymetrix Human Genome U133 Plus 2.0 Array), GSE48452 was based on GPL11532 (Affymetrix Human Gene 1.1 ST Array), GSE89632 was based on GPL14951 (Illumina HumanHT-12 WG-DASL V4.0 R2 expression bead chip), and GSE17470 was based on GPL2895 (GE Healthcare/Amersham Biosciences CodeLink Human Whole Genome Bioarray). All data were publicly available, and the present study did not involve any human or animal experimentation.

\subsection{Identification of differentially expressed genes}

To identify differentially expressed genes (DEGs), each dataset was analyzed by limma R package in Bioconductor, utilized to mine statistically significant DEGs based on the difference in their expression values between NASH and control samples [13]. Significant differential expression values were determined as a $\mid$ log fold change $\mid \geq 1$ and the adjusted P-value threshold of 0.05 .

\subsection{Integration of Microarray Data}

In this study, we considered microarray datasets consist of mRNA samples for our analysis. In this regard, datasets meeting the following criteria were excluded: (1) No declaration of disease stage or shortcomings in some samples; (2) Datasets included ambiguous samples; (3) Datasets which did not include any genes with adj-P-value <0.05 and the $|\log \mathrm{FC}|>1$. After analyzing each dataset separately and obtaining the important genes for each dataset, we can use the robust rank aggregation (RRA) method to combine our results and have a more clean and less noisy outcome. Moreover, this method helped us gain a general idea about our result and focus better on the important genes. Therefore, we used the RRA method to aggregate up- and down-regulated gene lists after analyzing each dataset [14]. Genes with P-value $<0.05$ and the $|\log \mathrm{FC}|>1$ were considered as significant.

\subsection{Construction of Protein-Protein Interaction}

For constructing a Protein-Protein Interaction (PPI) network, we inserted integrated DEGs to the STRING (http://www.string-db.org/), which is a biological database and web resource for known and predicted PPIs [15]. In order to plot the neutral interactive network, the confidence parameter was considered 0.400 (Medium Confidence).

\subsection{Identification of Co-expression network and hub genes}

To recognize the Co-expression network and hub genes, the outcome of the STRING database was reconstructed with Cytoscape software (version 3.7.0) [16]. The Molecular Complex Detection (MCODE) [17] and CytoHubba [18] plug-ins of Cytoscape were used to identify the co-expression modules and hub genes, respectively. The criteria for using MCODE were set as: Degree Cutoff $=2$, Node Score Cutoff $=0.2, \mathrm{~K}-\mathrm{Core}=2$, and Max. Depth $=100$. Also, we used the Maximal Clique Centrality (MCC) method for Cytohubba.

\subsection{Gene Set Enrichment Analysis (GSEA)}

Gene Set Enrichment Analysis (GSEA) is a useful approach for interpreting gene expression data based on the functional annotation of DEGs. We can identify specific biological processes, molecular function, cellular component, and metabolic pathways by GSEA.

GSEA for DEGs was performed by Enrichr bioinformatics tool (https://maayanlab.cloud/Enrichr/) [19], to identify pathways from Kyoto Encyclopedia of Genes and Genomes (KEGG) 2019, WikiPathways 2019, and Reactome 2016, in addition to Gene Ontology (biological process (BP), molecular function (MF), and cellular component (CC)) enrichment analysis. The cut-off criterion for GSEA was adj-P-value $<0.05$. 


\section{Results:}

\subsection{Dataset extraction and identification of NASH-related genes}

In this study, gene expression microarray data were included 101 individuals (49 healthy controls and 52 with NASH), which were obtained from 4 different datasets, summarized in Table 1. to show statistical significance (adjP-value) versus magnitude of change (gene expression fold change) in each dataset, volcano plot was used (Fig 1). Volcano plot displaying differential expressed genes between NASH patients and healthy controls in different datasets. It plots significance versus fold-change, and by visualizing the gene expression analysis results, it helps us to better identify the genes with large fold-changes which are also statistically significant and have the potential to be biologically significant. After screening gene expression data according to the mentioned criteria (adj-P-value $<0.05,|\log \mathrm{FC}|>1)$ from each dataset, in order to integrate collecting candidate genes from each dataset, we used RRA analysis that make a ranking list based on adj-P-value. After removing duplicate data, the obtained list contains 119 down- and 260 up-regulated genes (Table1).

\subsection{PPI network construction, module analysis, and selection of hub genes}

To investigate the relationship between genes and their ranking in terms of the presence of this disease, a ProteinProtein Interaction (PPI) network was drawn in which each node represents a gene. Notably, genes that had disconnected from main networks were removed. Out of 379 nodes (all up- and down-regulated genes) in the network, in order to identify the hub genes, we used the Cytohubba plugin and selected the top 10 genes based on the degree and betweenness centrality measures (Fig 2). Finally, according to the highest score, we chose the top 4 modules, using MCODE (score >3). Module 1 includes 24 nodes and 110 edges with a cluster score of 9.565; Module 2, 22 nodes, 98 edges with score 9.333; Module 3, 4 nodes, 6 edges with score 4; Module 4, 16 nodes, 28 edges with score 3.733 (Fig 2).

\subsection{Functional and pathway enrichment analyses}

Due to the importance of upstream and downstream genes and possible biological pathways in the occurrence of NASH disease, pathway analysis was performed. So, three databases, such as KEGG 2019 Human, WikiPathways 2019 Human, and Reactome 2016, were used for the Pathway Analysis of Identified DEGs. The top 10 pathways related to both up- and down-regulated DEGs were summarized in Figure 3. Up-regulated genes were significantly enriched in cytokine-related pathways, including TGF- $\beta$, IL17, TNF, and oncostatin M, as well as Nuclear receptors, vitamin D and vitamin 12 pathways (Fig 3A). Down-regulated genes were enriched mostly in Gluconeogenesis, cholesterol biosynthesis, and gene regulation by SREBF (Fig 3B).

According to the datasets analyzed and the hub genes extracted, by considering the adj-P-value $<0.05$, we obtained the top 10 Biological Process and Molecular Functions which were summarized in Table 2. No Cellular component term was significant in our GO analysis for hub genes. For BP, hub genes were mostly enriched in cytokinemediated terms, inflammatory processes, response to lipopolysaccharide, and regulating DNA transcription. In MF analysis, hub genes were mainly associated with cytokine and chemokine activity, transcription factor and DNA binding, RNA polymerase II activity, and R-SMAD binding (Table 2).

\section{Discussion}

Non-alcoholic fatty liver disease (NAFLD) is the most common chronic liver disease, ranging from simple steatosis (fatty liver) to non-alcoholic steatohepatitis (NASH), hepatic fibrosis, cirrhosis, and liver cancer (HCC) [20]. NASH is the aggressive form of NAFLD, characterized by steatosis, cell injury, inflammation, and hepatocyte ballooning [21]. Although liver biopsy is the gold standard to diagnose NASH and fibrosis, it has some limitations and risks 
which prevent it from being used in the early stages of the disease. Therefore, exploring the molecular mechanisms of NASH is pivotal to improve early diagnosis.

In the present study, we used integrated bioinformatic methods for transcriptomic analysis. So, four microarray datasets (GSE89632, GSE37031, GSE17470, GSE48452) were analyzed, and a total of 379 DEGs, including 260 up- and 119 down-regulated genes, were identified and integrated by the RRA algorithm. Using STRING and Cytoscape, we constructed a PPI network for DEGs, and the MCODE plugin performed cluster analysis. Finally, 10 hub genes associated with NASH were obtained via the Cytohubba plugin, including JUN, IL-6, FOS, EGR1, CCL2, SERPINE1, CXCL8, PTGS2, IL1B, and MYC. All hub genes were among up-regulated DEGs and demonstrated the importance of these genes.

Feng et al. proved the significance of FOS as a hub gene in NAFLD/NASH diseases. It is demonstrated that CAT, ALDH2, and ALDH8A1 are associated with NASH progression to HCC [6]. A recent bioinformatics study has shown that IL-6, MYC, FOS, FOSB, and JUNB are among NASH hub genes, as well as IL-17 and TNF- $\alpha$ signaling pathways were enriched in KEGG analysis [11].

Our pathway analysis for up-regulated DEGs revealed that they are enriched in immune system pathways such as TGF- $\beta$, TNF- $\alpha$, IL-17, and oncostatin M signaling pathways. TGF- $\beta$ signaling regulates a wide range of cellular processes, such as proliferation, differentiation, migration, apoptosis, and specification, by binding to the serine/threonine kinases receptors on the cell surface [22]. It has been shown that increased TGF $\square \beta$ signaling in hepatocytes contributes to lipid accumulation by regulation of lipogenesis and $\beta \square$ oxidation $\square$ related gene expression. Also, TGF $\square \beta$ Induces cell death in steatotic hepatocytes through Smad and Rho/ROCK Signaling pathway, resulting in inflammation and fibrosis [23].

Our analysis demonstrated that FOS (c-Fos) is a crucial gene in the NASH and is involved in the signaling pathways, including TGF $\square \beta$, IL-17, TNF- $\alpha$, and oncostatin M. It has been proved that TGF- $\beta$ stimulates c-Fos, and c-Fos expression in mice liver increases immune cell infiltration and inflammation [24, 25]. Also, TNF- $\alpha$-which is an essential mediator of inflammation- production will be elevated in LPS injected Fos-/- mice. So, FOS might act as an inflammatory response suppressor [26]. TNF- $\alpha$ is overexpressed in the liver and adipose tissues of obese patients, and the expression of TNF- $\alpha$ receptor-type 1 mRNA is higher in NASH patients' liver tissue [27]. Manco et al. researched on the serum levels of TNF- $\alpha$ in children with NAFLD and showed that TNF- $\alpha$ levels were significantly associated with a NAS score of 5 and more [28]. IL-17 and TNF- $\alpha$ pathways were also in PPI clusters 1 and 2, where IL-6 was enriched in both of them. Many studies have been reported the elevated levels of IL-6 and TNF- $\alpha$ expression in blood and serum samples of NAFLD patients, correlated with steatosis and NASH progression [29-31]. IL-6-deficient displayed less lobular inflammation and serum ALT than wild-type in MCD diet-induced NASH mice [32]. Conversely, some studies reported that the blocked IL-6 signaling or IL-6-deficient in MCD or HFD diet-fed mice results in steatosis and inflammation, demonstrating the protective role of IL-6 against the NASH development $[33,34]$. Association of IL- 6 with TGF $\square \beta$ promotes Th17 expression, which produces IL-17, a proinflammatory cytokine, inducing the production of other cytokines such as TGF $\square \beta$ and TNF- $\alpha$. Therefore, it has been implicated in liver inflammation and fibrosis progression by activation of Kupffer cells. It has been reported that IL $\square 17$ mRNA expression was increased in NASH patients compared with healthy controls and plays a pivotal role in hepatocyte steatosis and hepatic inflammation associated with NASH [35]. Besides, it has a fibrogenic effect by stimulating Kupffer cells to express cytokines, like IL-6, IL-1, TNF- $\alpha$, and TGF $\square$, and stimulating hepatic stellate cells to express collagen-1 and transformation to myofibroblasts by Stat3 [36]. There are some reports about the relation between IL-17 and adipogenesis. It suppresses adipogenesis by inhibiting expression of several proadipogenic transcription factors, including PPAR- $\gamma$, C/EBP $\alpha$, and KLF15, and enhances the expression of antiadipogenic TFs such as KLF2 and KLF3 [37]. PPAR- $\gamma$ and C/EBP $\alpha$ are the central regulators of lipogenesis, which induces adipocyte-specific genes, including fatty acid-binding protein 4 (FABP4), Glut4, and phosphoenolpyruvate carboxykinase (PEPCK) [38]. Also, PPAR- $\gamma$, which is elevated in the fatty liver, inhibits HSCs activation and fibrogenesis, and its deficiency protects against steatosis [39]. Vitamin D regulates the metabolism of free fatty acids by its effect on PPAR- $\gamma$ and relieving FFA-induced insulin resistance in vitro. Many studies have been found that 25(OH)D has lower levels in NAFLD/NASH patients than healthy controls [40]. Ding et al. reported that treatment of CCL4-induced mice with calcipotriol, a vitamin D receptor agonist, decreased fibrotic marker expressions such as COL1A1, TGF- $\beta 1$, and TIMP-1 [41]. Additionally, adipose tissue can directly affect hepatic cells. Induction of 
visceral adipose tissue-derived exosomes into HepG2 and HHSteC cells increased TIMP-1 and integrin av $\beta 5$ and decreased MMP-7 and PAI-1 expression in HepG2 cells, and increased TIMP-1, TIMP-4, Smad-3, integrins $\alpha v \beta 5$ and $\alpha v \beta 8$, and MMP-9 expressions in HSCs. The effect of integrin $\alpha v \beta 5$ on fibrosis induction in other organs through the TGF $\square \beta$ pathway suggests the same function in the pathogenesis of NAFLD [42]. In addition to the critical role of IL-6 by itself, Oncostatin M (OSM), a member of the IL-6 cytokine family, synthesized by activated monocytes and macrophages, activated $\mathrm{T}$ cells, dendritic cells, neutrophils, and eosinophils. Some investigations show that OSM receptor $\beta$ increases in the hepatocytes of the mouse model of hepatic steatosis. OSMR $\beta$-deficient mice exhibit delayed hepatocyte proliferation and persistent liver necrosis after CCl4 exposure, resulting in elevation of MMP-9, suppression of TIMP $\square 1$ and TIMP $\square 2$, and matrix degradation. So, CCl4-induced liver damage worsens in OSMR $\beta-/-$ mice. Additionally, OSM is expressed much more during NASH than that during steatosis in mice $[43,44]$. A recent study revealed that OSM expression has increased in human liver specimens from NAFLD patients, especially in the NASH stage, and there was no up-regulation in simple steatosis [45]. Up-regulated genes were also enriched in vitamin B12 metabolism. A recent study showed that serum levels of B12 have a negative correlation with NASH severity, whereas another research reported no difference in serum vitamin B12 levels between NAFLD/NASH patients and healthy controls [46-48]. As a result, more studies are needed to investigate this issue.

In the case of down-regulated genes, the pathway analysis was mainly enriched in gluconeogenesis and steroids biosynthesis, such as cholesterol and gene expression by SREBF. Liver steatosis induction by HFD diet in mice resulted in a significant increase of liver and plasma free fatty acid and triglyceride levels and plasma ALT activities. Moreover, gene expression analysis reveals that genes encoding cholesterol biosynthesis enzymes, such as squalene epoxidase (SQLE) and NAD(P)-dependent steroid dehydrogenase-like (NSDHL) were down-regulated [49]. These results confirm our analysis, in which SQLE and NSDHL were enriched in the cholesterol, sterol, and SREBF pathways. Liu et al. performed RNA-seq analysis of NAFLD-HCC tumor tissues and showed that SQLE is overexpressed in NAFLD-induced HCC patients. Further investigations on Sqle transgenic mice demonstrated higher hepatocyte ballooning, inflammatory cell infiltration, and serum ALT and AST levels than wild type. Also, proinflammatory mediators mRNAs, including CCL-12, CCL-20, CCR-5, CXCL9, CXCR4, IL-2, IL-4, IL-18r1, OSM, and SPP1 were increased [50]. So, more studies are needed to find out the exact mechanism of SQLE in NASH. A recent study on the Hela cell line showed that SQLE up-regulation is regulated by SP1 and SREBF2 transcription factors, increasing cholesterol and cholesteryl ester accumulation [51].

We performed GO analysis for hub genes, and the results indicated that most of them are involved in biological processes such as 'cellular response to cytokine stimulus', 'cytokine-mediated signaling pathway', and 'inflammatory response via cytokine and chemokine activity' molecular functions. A new meta-analysis study revealed that the concentration of CCL2 and CXCL8 in the NAFL and CCL3, CCL4, CCL20, CXCL8, and CXCL10 in the NASH patients was higher than that in the control group, respectively. It has been found that CCl2 is secreted by activated HSCs and Kupffer cells, which contributed to the lipid accumulation in hepatocytes and increased macrophage accumulation in the liver and adipose tissue, leading to fatty liver. Also, activated HSCs and Kupffer cells secrete CXCL8, which has an important role in NASH by recruitment of neutrophils via activation of the AKT/mTOR/STAT3 pathway [52]. An in vitro study showed that TGF- $\beta 1$ significantly up regulated CCL2 and CXCL8 concentrations in HSCs cell line [53]. Gore et al. studied on the inflammation in an ex vivo NASH murine model and reported that the gene expression of IL-6, IL1B, and TNF- $\alpha$ was increased [54]. Interestingly, IL1B in association with IL-23 induced IL-17, IL-21, and IL-22 expression by gamma delta $(\gamma \delta)$ T cells in the absence of additional signals [55]. JUN and FOS are AP-1 transcription factor subunits, enriched in activating transcription factor binding molecular function. Some studies observed an increased level of c-Jun -the best-characterized AP1 component- in mouse models and human NAFLD patients, showing a correlation between the inflammatory level and hepatocytic c-Jun expression [56, 57]. Expression of c-Fos in hepatocytes leads to liver inflammation so that granulocytes forming the bulk of recruited immune cells. Also, c-Fos influences the LXR/RXR pathway and controlling hepatic cholesterol, which its high expression leads to reduced pathway activity and accumulation of total and free cholesterols. It has been reported that PPAR- $\gamma$ is responsible for decrease LXR/RXR pathway activity [25]. Our GO analysis revealed that MYC (c-Myc) performs many biological processes, especially in 'inflammatory pathways', and interacts with many other inflammation mediators such as IL- $1,2,4,8,10$, TNF- $\alpha$, NF- $\kappa$ B, PPAR- $\gamma$, 
and p53. IL-6-c-Myc and IL-6-AP-1-c-Myc pathways increase c-Myc expression, whereas IL-6-NF- $\kappa$ B-c-Myc pathway inhibits that. TGF- $\beta$ increases c-Myc expression via stimulating Smad3 and downregulates it by the Smad2 [58]. SERPINE1, also known as PAI-1, was enriched in 'response to lipopolysaccharide' BP. PAI-1 is a downstream target of TGF- $\beta$, assessed as an antithrombotic agent, and its plasma concentration is correlated with the increasing NASH/fibrosis stage [59, 60]. Augmented levels of PAI-1 have been considered as a risk factor for thrombosis and cardiovascular events by inhibiting tissue $\square$ type plasminogen activator (tPA) and urokinase plasminogen activator (uPA), the main enzymes for inducing fibrinolysis, results in fibrin accumulation [61]. Furthermore, this process occurs in alcohol $\square$ induced liver injury, in which ethanol causes PAI-1 activation, leading to fibrin accumulation in the liver by inhibiting its degradation. Fibrin deposition sensitizes the liver to LPS $\square$ induced necrosis and inflammation. [61, 62]. These findings link NAFLD/NASH to cardiovascular disease (CVD) and might elucidate the increased CVD risk associated with NAFLD.

\title{
5. Conclusion
}

In conclusion, by performing transcriptomic analyses on NASH datasets, our study illustrated the potential signaling pathways and processes that may play a critical role in the molecular pathogenesis of the disease. Our results suggest the crucial functions of inflammatory responses and adipogenesis through the significant role of TGF- $\beta$, TNF- $\alpha$, and IL-17, as well as the association of hub genes such as JUN, IL-6, FOS, CCL2, SERPINE1, IL1B, and MYC. These findings may serve as useful markers for future studies on NASH diagnostic and therapeutic.

\section{Acknowledgments}

The authors would like to thank the Research Institute of Gastroenterology and Liver Diseases of the Shahid Beheshti Medical University for its financial support of this study

\section{CONFLICT OF INTEREST}

The authors declare no competing financial interests.

\author{
Abbreviation: \\ NAFLD, Non-alcoholic fatty liver disease; NASH, non-alcoholic steatohepatitis; GEO, Gene Expression Omnibus; \\ DEGs, Differentially Expressed Genes; RRA, Robust Rank Aggregation; PPI, Protein-Protein Interaction; GSEA, \\ Gene Set Enrichment Analysis ; KEGG, \\ Kyoto Encyclopedia of Genes and Genomes; BP, Biological Process ; MF, Molecular Function ; \\ $\mathrm{CC}$, Cellular Component;
}




\section{References}

1. Xiong, X., et al., Landscape of intercellular crosstalk in healthy and NASH liver revealed by singlecell secretome gene analysis. Molecular cell, 2019. 75(3): p. 644-660. e5.

2. Diehl, A.M. and C. Day, Cause, pathogenesis, and treatment of nonalcoholic steatohepatitis. N Engl J Med, 2017. 377: p. 2063-2072.

3. Samji, N.S., et al., Liver transplantation for nonalcoholic steatohepatitis: pathophysiology of recurrence and clinical challenges. Digestive diseases and sciences, 2019: p. 1-18.

4. Agbim, U., et al., Impact of nonmalignant portal vein thrombosis in transplant recipients with nonalcoholic steatohepatitis. Liver Transplantation, 2019. 25(1): p. 68-78.

5. Caligiuri, A., A. Gentilini, and F. Marra, Molecular pathogenesis of NASH. International journal of molecular sciences, 2016. 17(9): p. 1575.

6. Feng, G., et al., Bioinformatics analysis reveals novel core genes associated with nonalcoholic fatty liver disease and nonalcoholic steatohepatitis. Gene, 2020: p. 144549.

7. Kutlu, O., H.N. Kaleli, and E. Ozer, Molecular pathogenesis of nonalcoholic steatohepatitis(NASH-) related hepatocellular carcinoma. Canadian Journal of Gastroenterology and Hepatology, 2018. 2018.

8. Elbadawy, M., et al., Efficacy of primary liver organoid culture from different stages of nonalcoholic steatohepatitis (NASH) mouse model. Biomaterials, 2020. 237: p. 119823.

9. Cui, H. and X. Zhang, Occurrence and clinical management of nonalcoholic fatty liver disease in obesity patients: a literature review. Journal of Pediatric Endocrinology and Metabolism, 2020. 33(5): p. 579-584.

10. Li, L., et al., Identification of key genes in non \#alcoholic fatty liver disease progression based on bioinformatics analysis. Molecular medicine reports, 2018. 17(6): p. 7708-7720.

11. Ye, J., et al., Integrated Multichip Analysis Identifies Potential Key Genes in the Pathogenesis of Nonalcoholic Steatohepatitis. Frontiers in endocrinology, 2020. 11.

12. Edgar, R., M. Domrachev, and A.E. Lash, Gene Expression Omnibus: NCBI gene expression and hybridization array data repository. Nucleic acids research, 2002. 30(1): p. 207-210.

13. Ritchie, M.E., et al., limma powers differential expression analyses for RNA-sequencing and microarray studies. Nucleic acids research, 2015. 43(7): p. e47-e47.

14. Kolde, R., et al., Robust rank aggregation for gene list integration and meta-analysis. Bioinformatics, 2012. 28(4): p. 573-580.

15. Szklarczyk, D., et al., STRING v11: protein-protein association networks with increased coverage, supporting functional discovery in genome-wide experimental datasets. Nucleic acids research, 2019. 47(D1): p. D607-D613.

16. Shannon, P., et al., Cytoscape: a software environment for integrated models of biomolecular interaction networks. Genome research, 2003. 13(11): p. 2498-2504.

17. Bader, G.D. and C.W. Hogue, An automated method for finding molecular complexes in large protein interaction networks. BMC bioinformatics, 2003. 4(1): p. 2.

18. Chin, C.-H., et al., cytoHubba: identifying hub objects and sub-networks from complex interactome. BMC systems biology, 2014. 8(S4): p. S11.

19. Kuleshov, M.V., et al., Enrichr: a comprehensive gene set enrichment analysis web server 2016 update. Nucleic acids research, 2016. 44(W1): p. W90-W97. 
20. Wree, A., et al., From NAFLD to NASH to cirrhosis-new insights into disease mechanisms. Nature reviews Gastroenterology \& hepatology, 2013. 10(11): p. 627-636.

21. Ratziu, V., et al., A position statement on NAFLD/NASH based on the EASL 2009 special conference. Journal of hepatology, 2010. 53(2): p. 372-384.

22. Shi, Y. and J. Massagué, Mechanisms of TGF-B signaling from cell membrane to the nucleus. cell, 2003. 113(6): p. 685-700.

23. Yang, L., et al., Transforming growth factor beta signaling in hepatocytes participates in steatohepatitis through regulation of cell death and lipid metabolism in mice. Hepatology, 2014. 59(2): p. 483-495.

24. Angel, P. and M. Karin, The role of Jun, Fos and the AP-1 complex in cell-proliferation and transformation. Biochimica et Biophysica Acta (BBA)-Reviews on Cancer, 1991. 1072(2-3): p. 129-157.

25. Bakiri, L., et al., Liver carcinogenesis by FOS-dependent inflammation and cholesterol dysregulation. Journal of Experimental Medicine, 2017. 214(5): p. 1387-1409.

26. Ray, N., et al., c-Fos suppresses systemic inflammatory response to endotoxin. International immunology, 2006. 18(5): p. 671-677.

27. Crespo, J., et al., Gene expression of tumor necrosis factor $\alpha$ and TNF-receptors, $p 55$ and p75, in nonalcoholic steatohepatitis patients. Hepatology, 2001. 34(6): p. 1158-1163.

28. Manco, M., et al., Correlation of serum TNF- $\alpha$ levels and histologic liver injury scores in pediatric nonalcoholic fatty liver disease. American journal of clinical pathology, 2007. 127(6): p. 954-960.

29. García-Galiano, D., et al., IL-6 and IGF-1 are independent prognostic factors of liver steatosis and non-alcoholic steatohepatitis in morbidly obese patients. Obesity surgery, 2007. 17(4): p. 493.

30. Khura, J., et al., Evaluation of Pro-Inflammatory Markers IL-6 and TNF-a and their Correlation with Non-Alcoholic Fatty Liver Disease. Journal of Advanced Research in Medicine (E-ISSN: 23497181 \& P-ISSN: 2394-7047), 2019. 6(2): p. 1-6.

31. Kugelmas, M., et al., Cytokines and NASH: a pilot study of the effects of lifestyle modification and vitamin E. Hepatology, 2003. 38(2): p. 413-419.

32. Mas, E., et al., IL-6 deficiency attenuates murine diet-induced non-alcoholic steatohepatitis. PloS one, 2009. 4(11): p. e7929.

33. Yamaguchi, K., et al., Blockade of IL-6 signaling exacerbates liver injury and suppresses antiapoptotic gene expression in methionine choline-deficient diet-fed $\mathrm{db} / \mathrm{db}$ mice. Laboratory investigation, 2011. 91(4): p. 609-618.

34. Matthews, V.B., et al., Interleukin-6-deficient mice develop hepatic inflammation and systemic insulin resistance. Diabetologia, 2010. 53(11): p. 2431-2441.

35. Tang, Y., et al., Interleukin-17 exacerbates hepatic steatosis and inflammation in non-alcoholic fatty liver disease. Clinical \& Experimental Immunology, 2011. 166(2): p. 281-290.

36. Meng, F., et al., Interleukin-17 signaling in inflammatory, Kupffer cells, and hepatic stellate cells exacerbates liver fibrosis in mice. Gastroenterology, 2012. 143(3): p. 765-776. e3.

37. Ahmed, M. and S.L. Gaffen, IL-17 inhibits adipogenesis in part via C/EBP $\alpha, P P A R Y$ and Krüppellike factors. Cytokine, 2013. 61(3): p. 898-905.

38. Rosen, E.D., et al., Transcriptional regulation of adipogenesis. Genes \& development, 2000. 14(11): p. 1293-1307.

39. Trauner, M. and E. Halilbasic, Nuclear receptors as new perspective for the management of liver diseases. Gastroenterology, 2011. 140(4): p. 1120-1125. e12.

40. Wang, X., et al., Association between vitamin $D$ and non-alcoholic fatty liver disease/nonalcoholic steatohepatitis: results from a meta-analysis. International journal of clinical and experimental medicine, 2015. 8(10): p. 17221. 
41. Ding, N., et al., A vitamin D receptor/SMAD genomic circuit gates hepatic fibrotic response. Cell, 2013. 153(3): p. 601-613.

42. Koeck, E.S., et al., Adipocyte exosomes induce transforming growth factor beta pathway dysregulation in hepatocytes: a novel paradigm for obesity-related liver disease. journal of surgical research, 2014. 192(2): p. 268-275.

43. Komori, T. and Y. Morikawa, Oncostatin $M$ in the development of metabolic syndrome and its potential as a novel therapeutic target. Anatomical Science International, 2018. 93(2): p. 169176.

44. Nakamura, K., et al., Hepatocyte proliferation and tissue remodeling is impaired after liver injury in oncostatin M receptor knockout mice. Hepatology, 2004. 39(3): p. 635-644.

45. Foglia, B., et al., Oncostatin M, A Profibrogenic Mediator Overexpressed in Non-Alcoholic Fatty Liver Disease, Stimulates Migration of Hepatic Myofibroblasts. Cells, 2020. 9(1): p. 28.

46. Mahamid, M., et al., Folate and B12 levels correlate with histological severity in NASH patients. Nutrients, 2018. 10(4): p. 440.

47. Polyzos, S.A., et al., Serum vitamin B12 and folate levels in patients with non-alcoholic fatty liver disease. International journal of food sciences and nutrition, 2012. 63(6): p. 659-666.

48. Kumar, S., S.F. Alam, and P.K. Ganguly, Obesity-Induced Non-alcoholic Fatty Liver Disease (NAFLD): Role of Hyperhomocysteinemia, in Pathophysiology of Obesity-Induced Health Complications. 2020, Springer. p. 181-192.

49. Kirpich, I.A., et al., Integrated hepatic transcriptome and proteome analysis of mice with high-fat diet-induced nonalcoholic fatty liver disease. The Journal of nutritional biochemistry, 2011. 22(1): p. 38-45.

50. Liu, D., et al., Squalene epoxidase drives NAFLD-induced hepatocellular carcinoma and is a pharmaceutical target. Science translational medicine, 2018. 10(437).

51. Zhang, C., et al., OSBPL2 deficiency upregulate SQLE expression increasing intracellular cholesterol and cholesteryl ester by AMPK/SP1 and SREBF2 signalling pathway. Experimental cell research, 2019. 383(2): p. 111512.

52. Pan, X., et al., Chemokines in Non-alcoholic Fatty Liver Disease: A Systematic Review and Network Meta-Analysis. Frontiers in immunology, 2020. 11: p. 1802.

53. $\mathrm{Xu}, \mathrm{X} . \mathrm{Y}$. , et al., Targeting Follistatin like 1 ameliorates liver fibrosis induced by carbon tetrachloride through TGF-81-miR29a in mice. Cell Communication and Signaling, 2020. 18(1): p. $1-12$.

54. Gore, E., et al., Investigating fibrosis and inflammation in an ex vivo NASH murine model. American Journal of Physiology-Gastrointestinal and Liver Physiology, 2020. 318(2): p. G336G351.

55. Sutton, C.E., et al., Interleukin-1 and IL-23 induce innate IL-17 production from $\gamma \delta T$ cells, amplifying Th17 responses and autoimmunity. Immunity, 2009. 31(2): p. 331-341.

56. Dorn, C., et al., Increased expression of c-Jun in nonalcoholic fatty liver disease. Laboratory Investigation, 2014. 94(4): p. 394-408.

57. Schulien, I., et al., The transcription factor c-Jun/AP-1 promotes liver fibrosis during non-alcoholic steatohepatitis by regulating Osteopontin expression. Cell Death \& Differentiation, 2019. 26(9): p. 1688-1699.

58. Liu, T., et al., Interactions between Myc and mediators of inflammation in chronic liver diseases. Mediators of inflammation, 2015. 2015.

59. Targher, G., et al., Plasma PAl-1 levels are increased in patients with nonalcoholic steatohepatitis. Diabetes care, 2007. 30(5): p. e31-e32.

60. Mukherjee, S., et al., Development and validation of an in vitro 3D model of NASH with severe fibrotic phenotype. American journal of translational research, 2019. 11(3): p. 1531. 
61. Barrera, F. and J. George, Prothrombotic factors and nonalcoholic fatty liver disease: An additional link to cardiovascular risk? Hepatology, 2014. 59(1): p. 16-18.

62. Beier, J.I., et al., Fibrin accumulation plays a critical role in the sensitization to lipopolysaccharide-induced liver injury caused by ethanol in mice. Hepatology, 2009. 49(5): p.

\begin{tabular}{ccc}
\hline \hline Biological Process & Adj-P-value & Genes \\
\hline \hline
\end{tabular}

1545-1553.

Table 1. Characteristics of included $(\checkmark)$ and excluded $(x)$ microarray datasets used for this study

\begin{tabular}{|c|c|c|c|c|c|c|c|}
\hline Dataset ID & Platform & Country & year & $\begin{array}{l}\text { Control } \\
\text { Samples }\end{array}$ & $\begin{array}{c}\text { NASH } \\
\text { Samples }\end{array}$ & $\begin{array}{c}\text { Up- } \\
\text { Regulated } \\
\text { Genes Count }\end{array}$ & $\begin{array}{c}\text { Down- } \\
\text { Regulated } \\
\text { Genes count }\end{array}$ \\
\hline$\checkmark$ GSE37031 & GPL14877 & Spain & 2013 & 7 & 8 & 93 & 65 \\
\hline$\checkmark$ GSE48452 & GPL11532 & Germany & 2016 & 14 & 18 & 32 & 24 \\
\hline$\checkmark$ GSE89632 & GPL14951 & Canada & 2017 & 24 & 19 & 124 & 34 \\
\hline$\checkmark$ GSE17470 & GPL2895 & USA & 2014 & 4 & 7 & 63 & 46 \\
\hline × GSE83452 & GPL16686 & France & 2019 & & & \multirow{7}{*}{-- } & \\
\hline x GSE68421 & GPL16686 & Denmark & 2019 & & & & \\
\hline x GSE24807 & GPL2895 & USA & 2019 & & & & \\
\hline × GSE96971 & GPL14951 & Canada & 2019 & & & & \\
\hline × GSE49012 & GPL17470 & USA & 2013 & & & & \\
\hline × GSE72756 & GPL16956 & China & 2018 & & & & \\
\hline × GSE134438 & GPL16686 & Japan & 2019 & & & & \\
\hline
\end{tabular}




\begin{tabular}{|c|c|c|}
\hline cellular response to cytokine stimulus & $1.52 \mathrm{E}-08$ & EGR1; IL6; CXCL8; IL1B; MYC; CCL2; FOS; PTGS2 \\
\hline cellular response to oxygen-containing compound & $2.48 \mathrm{E}-08$ & EGR1; IL6; JUN; CXCL8; SERPINE1; CCL2; FOS \\
\hline cytokine-mediated signaling pathway & $6.98 \mathrm{E}-08$ & EGR1; IL6; CXCL8; IL1B; MYC; CCL2; FOS; PTGS2 \\
\hline cellular response to lipopolysaccharide & $5.51 \mathrm{E}-07$ & IL6; CXCL8; IL1B; SERPINE1; CCL2 \\
\hline response to lipopolysaccharide & $6.53 \mathrm{E}-06$ & IL6; JUN; CXCL8; SERPINE1; CCL2 \\
\hline positive regulation of cellular biosynthetic process & $6.19 E-06$ & EGR1; IL6; IL1B; MYC; PTGS2 \\
\hline positive regulation of acute inflammatory response & $2.98 \mathrm{E}-05$ & IL6; IL1B; PTGS2 \\
\hline $\begin{array}{l}\text { positive regulation of nucleic acid-templated } \\
\text { transcription }\end{array}$ & $2.98 \mathrm{E}-05$ & EGR1; IL6; JUN; IL1B; MYC; FOS \\
\hline cellular response to molecule of bacterial origin & $3.38 \mathrm{E}-05$ & IL6; CXCL8; SERPINE1; CCL2 \\
\hline inflammatory response & $3.73 \mathrm{E}-05$ & IL6; CXCL8; IL1B; CCL2; FOS \\
\hline Molecular Function & Adj-P-value & Genes \\
\hline cytokine activity & $8.09 \mathrm{E}-04$ & IL6; CXCL8; IL1B; CCL2 \\
\hline activating transcription factor binding & 0.002551997 & JUN; MYC; FOS \\
\hline $\begin{array}{l}\text { transcriptional repressor activity, RNA polymerase } \\
\text { II activating transcription factor binding }\end{array}$ & 0.005682222 & JUN; FOS \\
\hline transcription regulatory region DNA binding & 0.006651417 & EGR1; JUN; MYC; FOS \\
\hline R-SMAD binding & 0.008816878 & JUN; FOS \\
\hline $\begin{array}{l}\text { RNA polymerase II regulatory region sequence- } \\
\text { specific DNA binding }\end{array}$ & 0.009967739 & EGR1; JUN; MYC; FOS \\
\hline chemokine activity & 0.037845114 & CXCL8; CCL2 \\
\hline $\begin{array}{l}\text { RNA polymerase Il activating transcription factor } \\
\text { binding }\end{array}$ & 0.034577015 & JUN; FOS \\
\hline $\begin{array}{l}\text { RNA polymerase II core promoter proximal region } \\
\text { sequence-specific DNA binding }\end{array}$ & 0.031861736 & JUN; MYC; FOS \\
\hline chemokine receptor binding & 0.030076527 & CXCL8; CCL2 \\
\hline
\end{tabular}

Table 2. Top $10 \mathrm{GO}$ enrichment analysis for hub genes. No terms were significantly enriched in the 'cellular component'. 
bioRxiv preprint doi: https://doi.org/10.1101/2021.09.10.459743; this version posted September 11, 2021. The copyright holder for this preprint (which was not certified by peer review) is the author/funder, who has granted bioRxiv a license to display the preprint in perpetuity. It is made available under aCC-BY-NC-ND 4.0 International license.
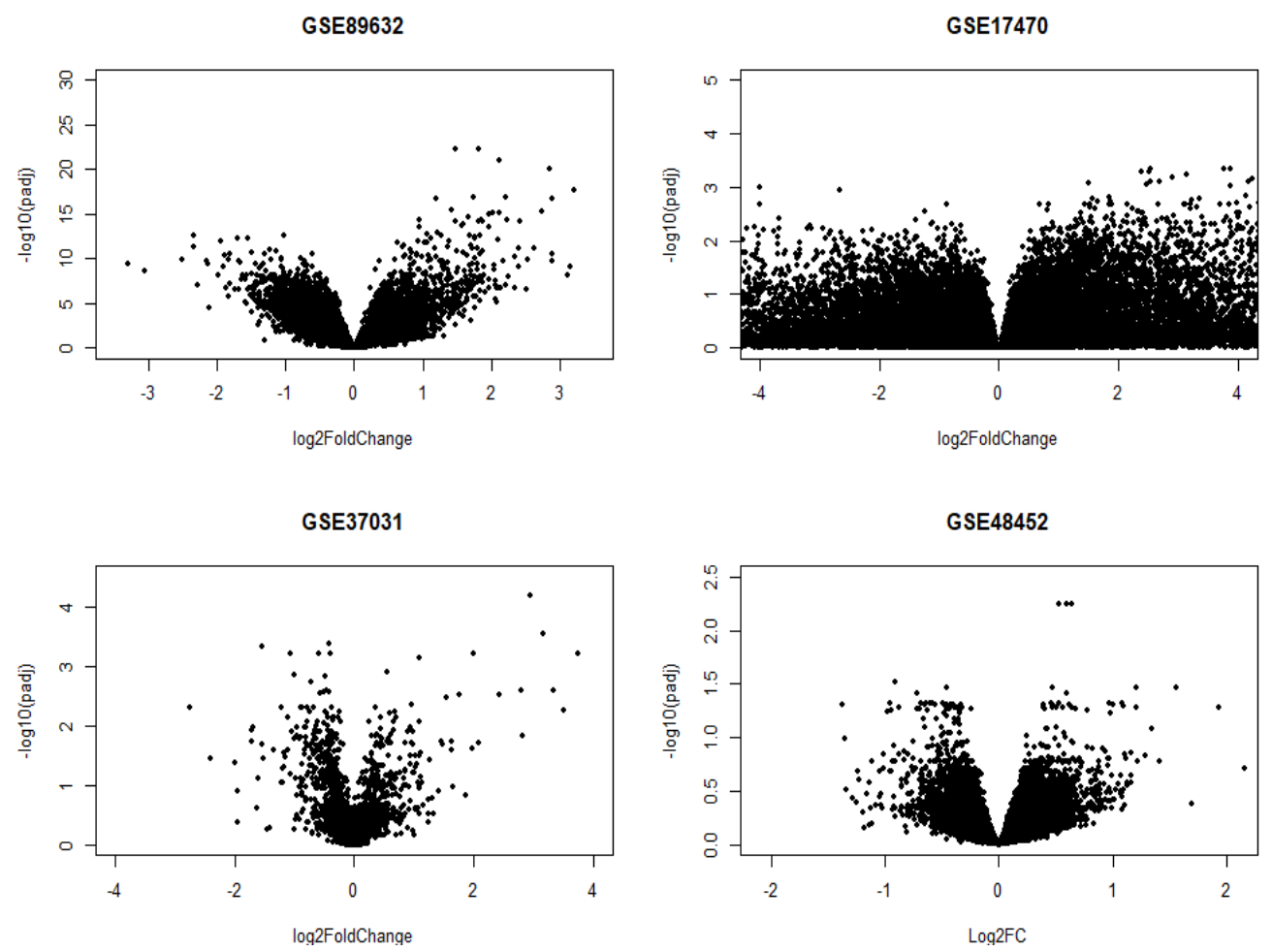

Fig 1. Volcano plots displaying differential expressed genes between NASH patients and healthy controls in different datasets. The vertical axis shows the expression adj-p-value of log 10 , and the horizontal axis indicates the log2-fold change value. 


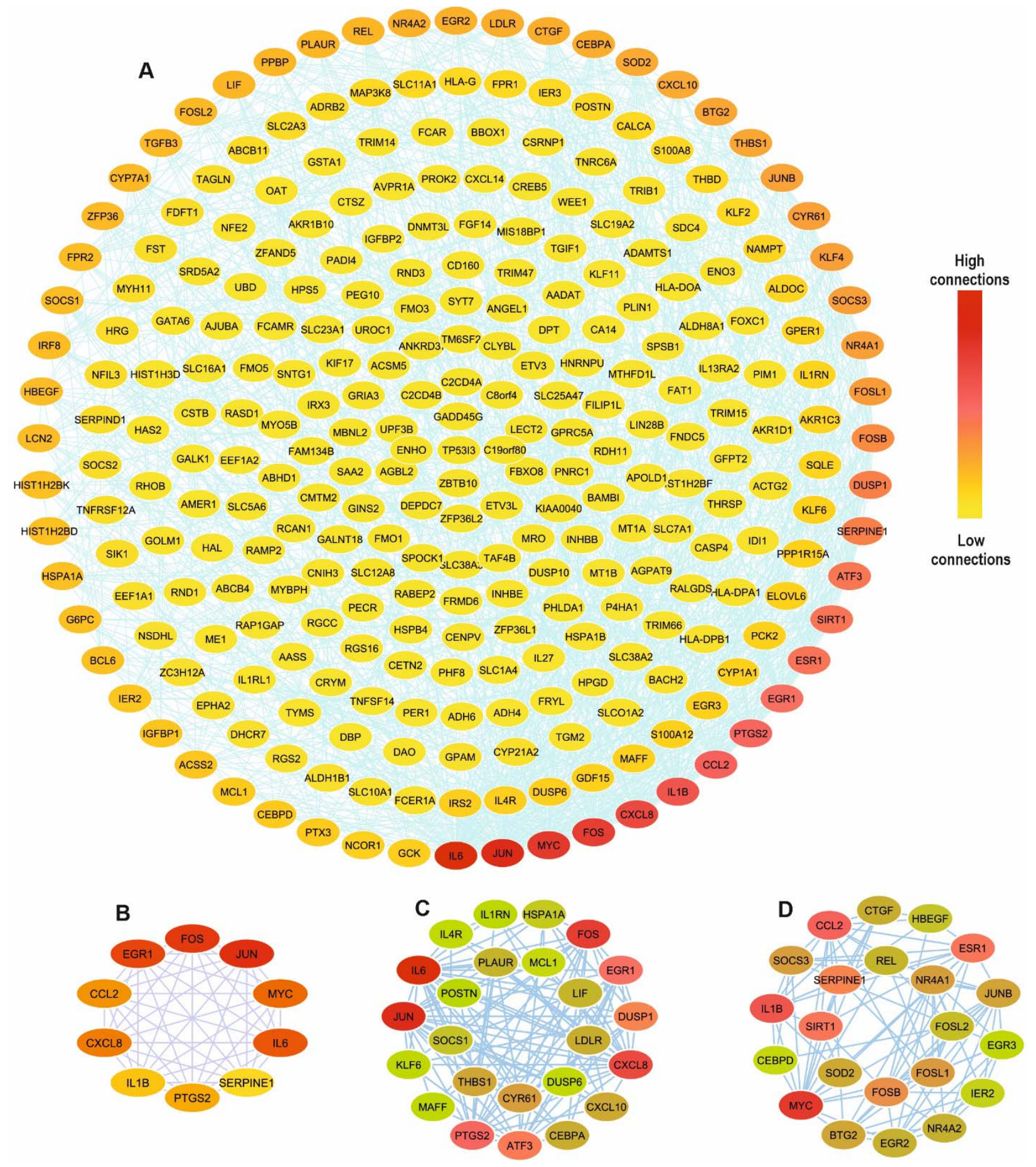

Fig 2. The PPI network analysis of NASH-related genes, containing GSE37031, GSE48452, GSE89632, GSE17470 datasets. (A) The overall PPI network of integrated DEGs, including 296 nodes and 1245 edges, (B) The top ten hub genes, (C) and (D) first two modules, derived from the overall PPI network. Red color indicates the high connections, and yellow color indicates the low ones. 
bioRxiv preprint doi: https://doi. org/10.1101/2021.09.10.459743. this version posted September 11, 2021. The copyright holder for this preprint (which was not certified by peer review) is the author/funder, who has granted bioRxiv a license to display the preprint in perpetuity. It is made available under aCC-BY-NC-ND 4.0 International license.
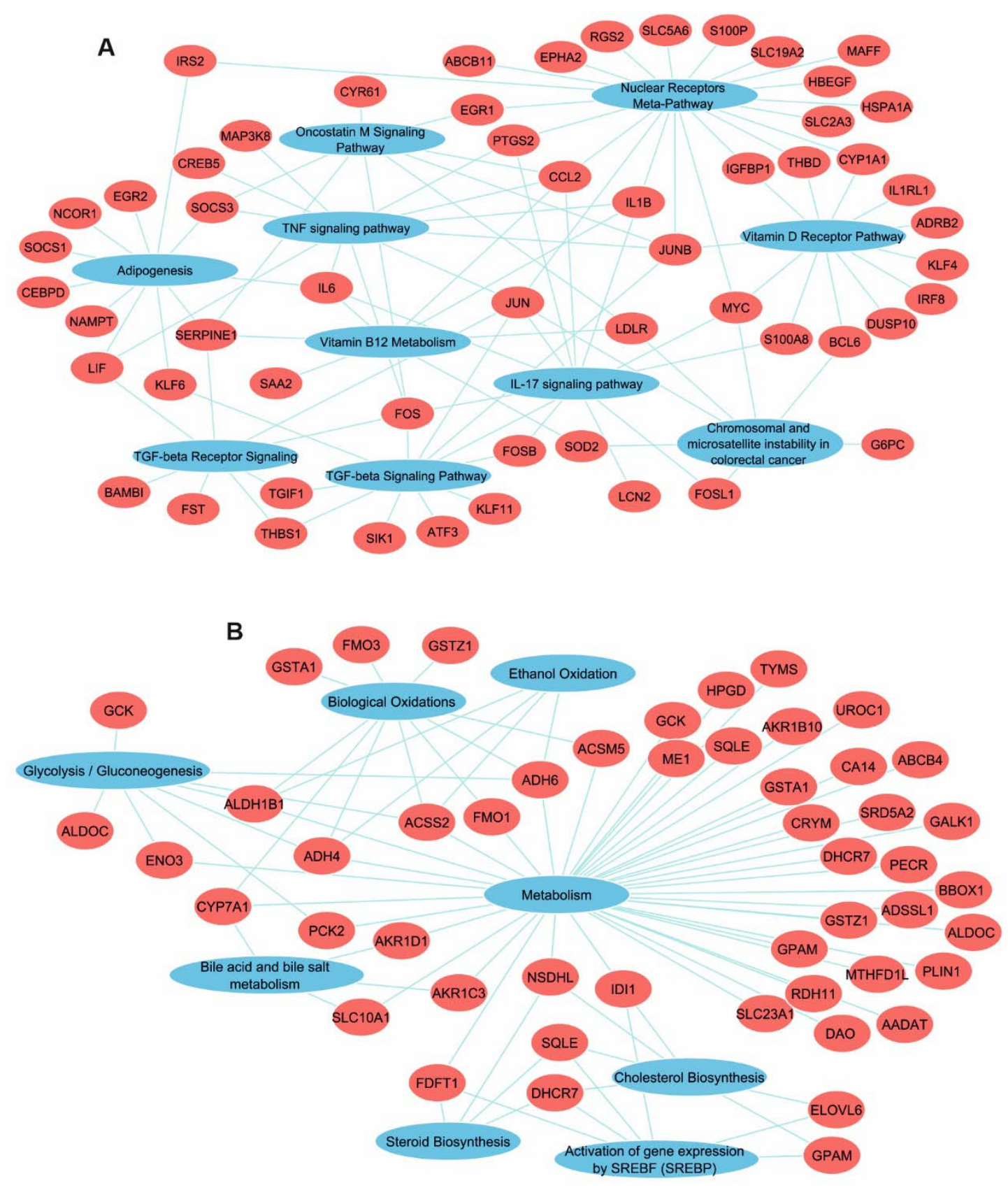

Fig 3. Pathway enrichment analysis for up- (A) and down-regulated (B) genes, using KEGG, Reactome, and Wikipathways databases 


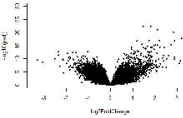

\begin{tabular}{ll} 
& \\
\hdashline & -1 \\
-1 & -1 \\
-1 & -1 \\
-1 & -1
\end{tabular}
GSE270\$1

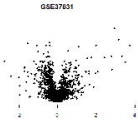

Isqa $\times 5$ Son:s

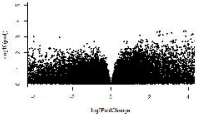

GSE $48 \angle 62$
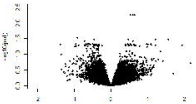

$1=126$ 

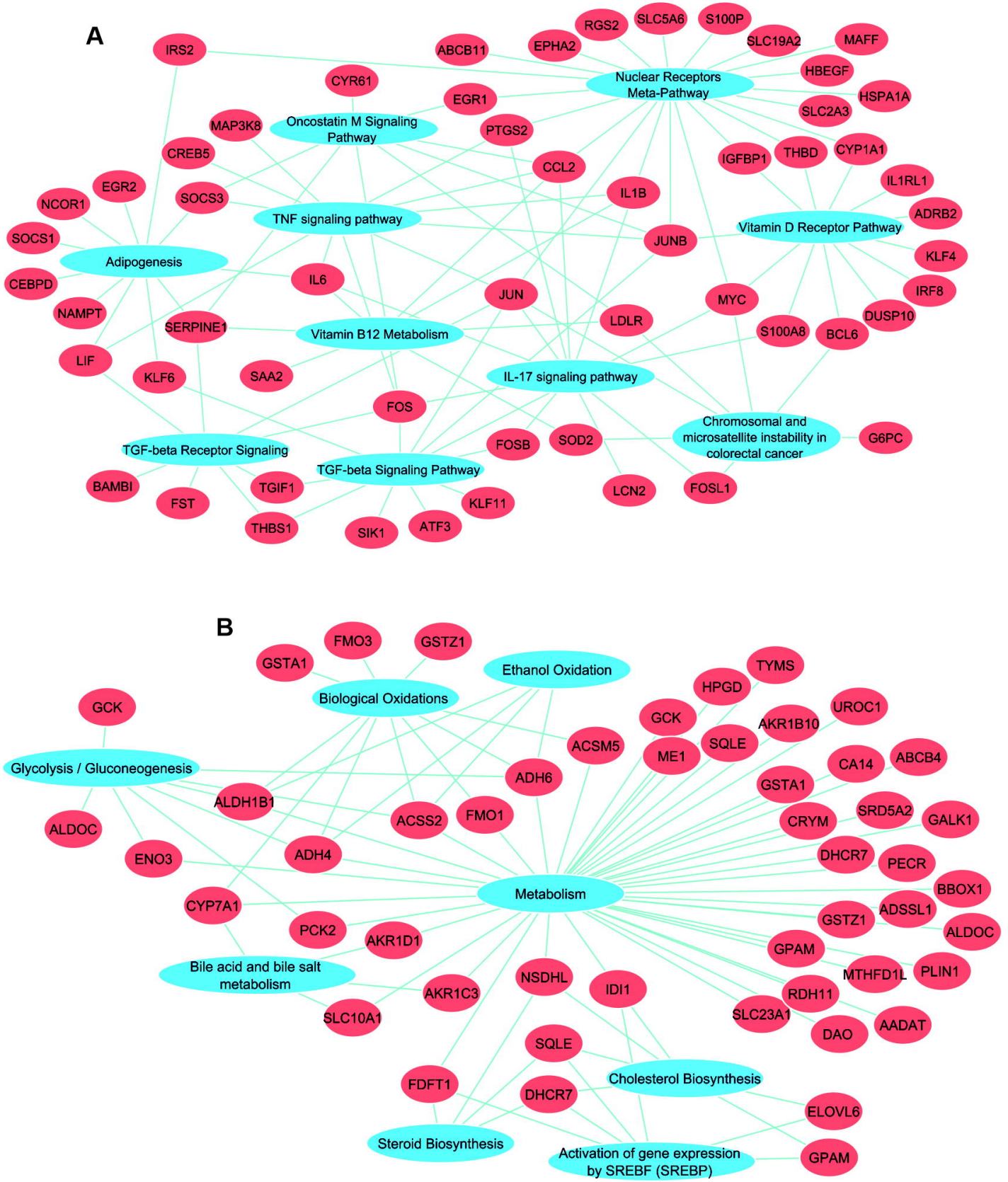


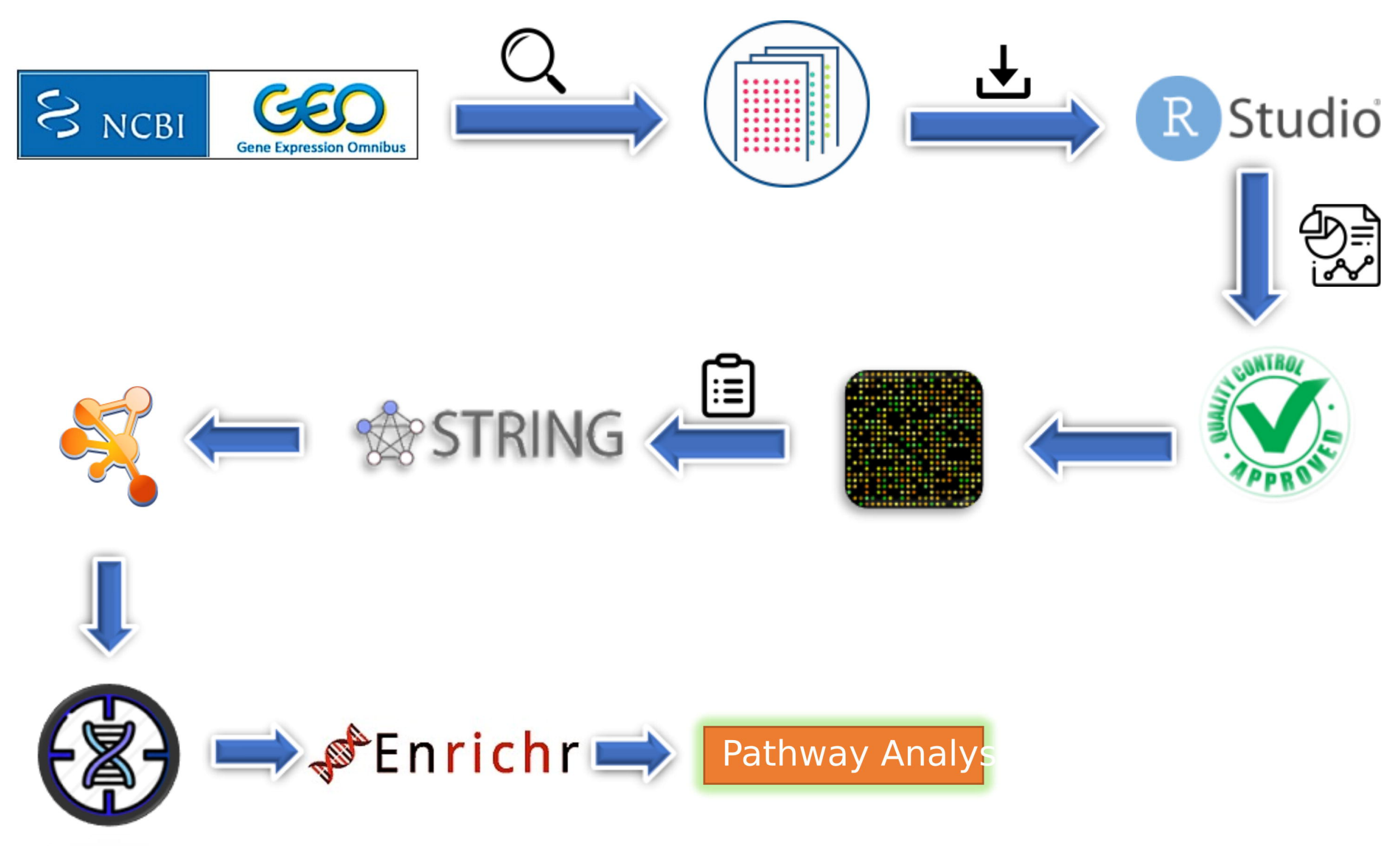

